\title{
Phronesis
}

\section{L’apprentissage professionnel par compétence} Analyse comparée entre la démarche théorique et la pratique sur le terrain

\section{The Professional Learning by Competence Comparison Analysis between the Theoretical Approach and the Practical}

\section{Zoualfakar Jammoul}

Volume 2, numéro 4, octobre 2013

Du bon usage de la notion de « professionnalisation " : entre modèle de formation, élaborations des politiques et attentes des acteurs; quelles convergences?

URI : https://id.erudit.org/iderudit/1022258ar

DOI : https://doi.org/10.7202/1022258ar

Aller au sommaire du numéro

Éditeur(s)

Institut de recherche sur les pratiques éducatives

ISSN

1925-4873 (numérique)

Découvrir la revue

Citer cet article

Jammoul, Z. (2013). L’apprentissage professionnel par compétence : analyse comparée entre la démarche théorique et la pratique sur le terrain. Phronesis, 2(4), 18-36. https://doi.org/10.7202/1022258ar
Résumé de l'article

Dans les années 1980, l'introduction du concept de compétence dans les programmes d'apprentissage a fait l'objet d'une tentative expérimentale pour faire face à l'évolution des métiers. Ce concept a été appliqué dés la fin des années 1960 par les entreprises en vue d'augmenter leur efficience de production. Aujourd'hui, nous observons qu'un bon nombre de centres de recherche et d'apprentissage ainsi que des chercheurs continuent à travailler à l'amélioration des processus d'apprentissage basés sur le concept de compétence. La question qui se pose est de savoir quelles sont les limites d’adéquation de la démarche théorique aux réalités pratiques de terrain. d'utilisation que vous pouvez consulter en ligne.

https://apropos.erudit.org/fr/usagers/politique-dutilisation/ 


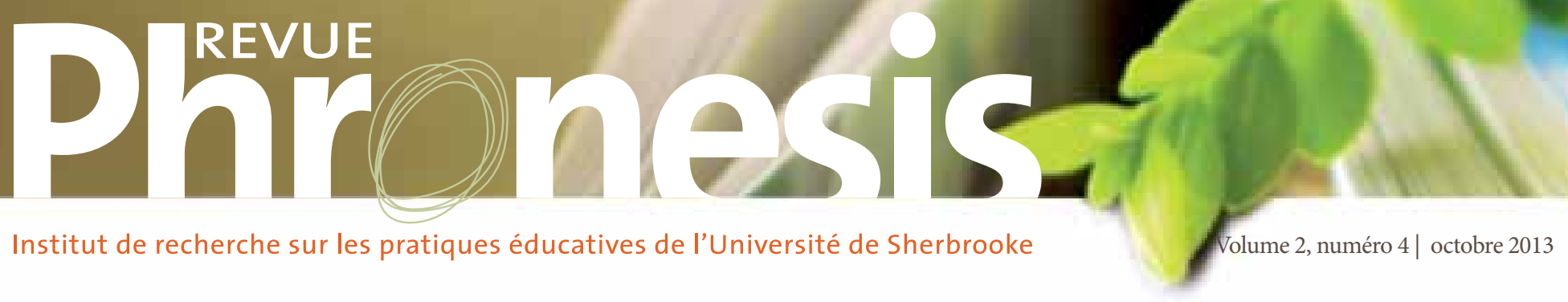

Institut de recherche sur les pratiques éducatives de l'Université de Sherbrooke

\title{
Lapprentissage professionnel par compétence Analyse comparee entre la démarche théorique et la pratique sur le terrain
}

\author{
Zoualfakar JAMMOUL
}

Laboratoire : UMR 5191 ICAR (Interactions, Corpus, Apprentissage, Représentations)

Université Lyon 2

86 rue Pasteur -69007 Lyon

zoualfakar@hotmail.fr

Mots clés : apprentissage, professionnel, compétence, comparaison, démarche, théorie, pratique.

Résumé : Dans les années 1980, l'introduction du concept de compétence dans les programmes d'apprentissage a fait l'objet d'une tentative expérimentale pour faire face à l'évolution des métiers. Ce concept a été appliqué dés la fin des années 1960 par les entreprises en vue d’augmenter leur efficience de production. Aujourd'hui, nous observons qu'un bon nombre de centres de recherche et d'apprentissage ainsi que des chercheurs continuent à travailler à lamélioration des processus d'apprentissage basés sur le concept de compétence. La question qui se pose est de savoir quelles sont les limites d’adéquation de la démarche théorique aux réalités pratiques de terrain.

\section{Title: The Professional Learning by Competence: Comparison Analysis between the Theoretical Approach and the Practical}

Keywords: learning, professional competence, comparison approach, theory, practice.

Abstract : In the 1980, the introduction of the concept of competence in learning programs has been an experimental attempt to cope with changing business. This concept was applied once in the late 1960s by companies to increase their production efficiency. Today, we observe that many centers of research and learning as well as researchers continue to work to improve the learning process based on the concept of competence. The question is what are the adequacy limits of the theoretical approach to practical realities on the ground? 
L'introduction de la notion de compétence dans le domaine de l'apprentissage professionnel, qui fait l'objet de cet article, comprend une série d'étapes et de changements qui affectent le rôle de l'enseignant et de lélève dans le processus d’apprentissage. De ce fait, une réhabilitation des enseignants et un examen des éléments propres à l'environnement scolaire et d'apprentissage sont nécessaire. En effet, les outils, les moyens, lorganisation des horaires entre la pratique et la théorie et enfin les méthodes dévaluation doivent être en adéquation avec le nouveau concept. Certes, l'introduction du concept de compétence dans le processus d'apprentissage, selon Barbier, a conduit à parler d'un apprenant en face d'un problème ou d'une situation complexe. (Barbier, 1996, p.38). Pour Gillet, l'apprenant est placé face à un obstacle en raison du manque des éléments nécessaires, à un moment donné, pour résoudre le problème; cette situation étant perçue comme un apprentissage. (Gillet, 1997, p.121). Scallon considère que chaque problème doit faire appel à une multitude déléments appartenant au répertoire cognitif et affectif de lélève (Scallon, 2010, p.34); enfin, pour Morlaix, face à l'apprentissage par compétence lenseignant doit organiser des situations complexes, inventer des problèmes, proposer des énigmes ou des projets, il n’a plus le rôle de démonstrateur. Dans ces approches, lobjectif est d'inciter lélève à réfléchir, à analyser, à renforcer son autonomie et son initiative dans le but d'agir face à une situation problème telle qu'il a la probabilité de la rencontrer dans le monde professionnel. Or, l'introduction de cette nouvelle logique dans le référentiel de la formation professionnelle initiale du Ministère de l'Éducation nationale (MEN) suite à son émergence dans le monde professionnel pour répondre aux besoins des entreprises, suscite néanmoins une interrogation :

Pourquoi les enseignants au sein du lycée professionnel ne prennent-ils pas en considération le concept de compétence lors de la conception des méthodes d’apprentissage?

Pour aborder cette problématique, nous allons nous appuyer sur les résultats obtenus lors de notre recherche en vue dobtenir un grade de doctorat en sciences de léducation; recherche intitulée : «Formation professionnelle et marché du travail, Étude exploratoire des interactions entre compétences scolaires et attentes du secteur de la restauration (technologie culinaire) à partir des représentations des acteurs ».

La formation professionnelle initiale, qui représente le cadre général de cette recherche, consiste par définition " à amener l’individu au niveau souhaité de performance ou de comportement par l'éducation et la pratique en lui apportant l'expérience et la théorie ». (T-KIT, 2004, p.11).

En France, le système de formation initiale est construit sur six niveaux :

- Le premier et le deuxième correspond au niveau BAC+3 ou +4

- Le troisième niveau correspond au niveau $\mathrm{BAC}+2$

- Le quatrième niveau correspond au niveau BAC

- Le cinquième correspond au niveau BEP ou CAP

- Le sixième niveau n’exige pas un niveau d’études. (Apprentissage et enseignement professionnel jusqu’en 2004 : sigles et définitions, 2005).

La formation initiale comprend deux branches, l’une générale et l'autre professionnelle. Cette dernière intègre des cours théoriques et des stages en entreprise chaque année d'une durée totale de 12 semaines afin de faire le lien entre le scolaire et l'activité professionnelle envisagée.

Pour notre recherche, nous nous sommes focalisés sur les classes de niveau 4 et 5 (BEP1, BEP2, BAC1 et BAC2); point de départ de la formation. En effet, nous avons choisi la formation initiale professionnelle car elle représente le maillon principal entre le monde scolaire et le monde professionnel. Par ailleurs, le choix du secteur « hôtellerie-restauration » a été établi pour deux raisons : la première étant le poids de ce dernier dans léconomie française où il contribue à faire de la France l'un des premiers acteurs mondiaux du tourisme. (FAFIH, 2011, p.3). Il est l'un des secteurs les plus dynamiques en termes de création d’emplois, le $4^{\text {ème }}$ employeur privé en France. (FAFIH, 2011). Cependant, il a la caractéristique d’avoir l'un des taux les plus élevés en terme de difficulté de recrutement (Enquête Besoins en Main 
d'œuvre, 2012). Deuxièmement, le choix de ce secteur est lié aussi à mon expérience en tant que travailleur saisonnier dans la restauration lors de mes études où j’ai pu observer que les jeunes diplômés nouvellement embauchés ne peuvent pas travailler sans la démonstration et les conseils du chef ou la supervision de l’un des travailleurs possédant déjà une expérience dans le domaine.

Cette situation paradoxale a orienté notre réflexion afin de comprendre pourquoi un secteur porteur souffre d'un manque de maind’œuvre alors que la formation initiale professionnelle fournit un vivier conséquent de diplômés. Pour aborder la thématique de cet article, nous allons décrire les démarches par compétence ainsi que la notion de situation problème qui est au cœur même de cette démarche. Puis, nous allons expliciter la méthodologie, les résultats obtenus et leur interprétation.

\section{La démarche d’apprentissage par compétence}

Nous ne pouvons parler de l’apprentissage sans aborder les démarches d’apprentissage par compétence. En effet, nos lectures ont révélé plusieurs démarches développées dans différentes disciplines (didactique disciplinaire et/ou professionnelle) qui correspondent au concept de compétence. Par exemple, Pastré met l'accent sur l'analyse du travail en didactique professionnelle (Pastré, 2002). Cette dernière se concentre sur l'analyse du contenu enseigné. Or, notre présence sur le terrain a été limitée à une observation externe, ce qui nous a amenés par la suite à opter pour la démarche de Guy Le Boterf pour être la référence théorique de notre recherche. En effet, cette démarche correspond plus au monde professionnel et elle se concentre sur le déroulement du processus d'apprentissage. La deuxième démarche retenue est celle mise en ouvre au sein de l'Institut Universitaire de Formation des Maitres (IUFM) de Rouen et qui est dispensée aux enseignants dans le cadre de leur formation pédagogique. Elle va nous servir de base de comparaison entre les démarches théoriques et celle dispensée en classe.

\subsection{La démarche de Guy Le Boterf}

La démarche de Guy Le Boterf comprend quatre étapes principales pour l'apprentissage d'une tâche complexe en vue de la construction des compétences :

1. Le moment de l'expérience vécue : action du sujet dans une situation donnée, mise en application des savoirs;

2. Le moment de l'explication : verbalisation et description de l'action réalisée permettant au sujet une mise à distance, un début de réflexion menant à une représentation de l'action;

3. Le moment de la conceptualisation : élaboration d'un savoir pragmatique fondé sur la construction de schèmes opératoires, d'une structure conceptuelle répondant à différentes situations;

4. Le moment du transfert ou de la transposition : mise à lépreuve des schèmes opératoires face à une nouvelle situation ce qui implique une remise en cause des représentations afin de reconstruire cette adéquation. (Le Boterf, 2007, p. 117-118).

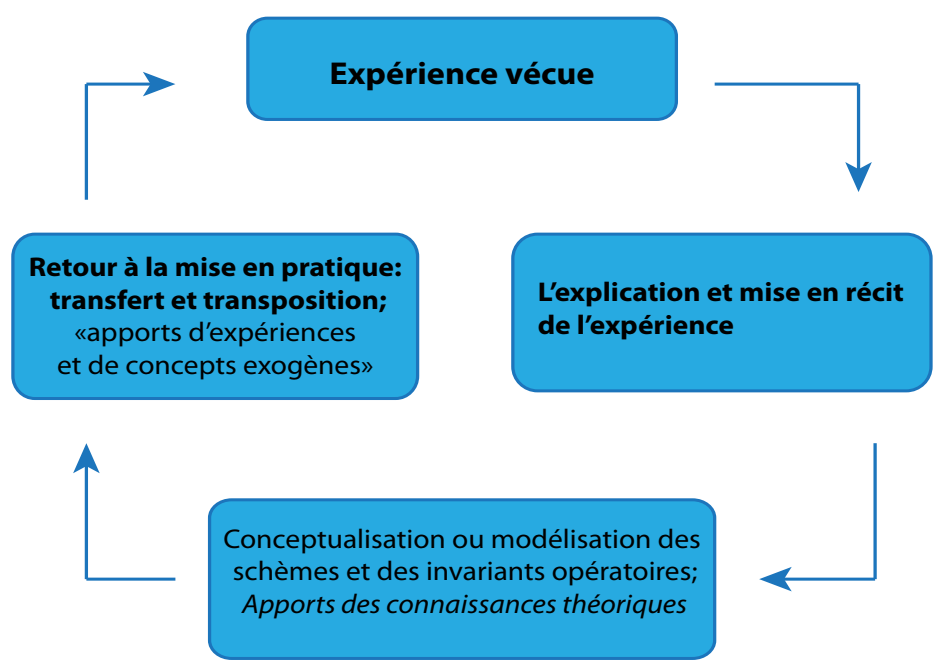

Figure 1 : La boucle d'apprentissage « expérientielle » de Le BOTERF, adaptée de Kolb (1984) et Piaget (1977); (Source: Conard, P., \& Devin, B. 2007). 
Cette démarche met l'apprenant face à une situation problème, ce qui suppose qu'il possède déjà une gamme de connaissances théoriques et pratiques qui lui permet d’aborder le problème rencontré tant par des processus manuels que des essais mentaux. Cette démarche convient plus aux professionnels quaux élèves qui ont besoin en premier d'acquérir une gamme de compétences théoriques et pratiques par un médiateur avant de passer à l'étape de l'apprentissage par situation problème. C'est ce que nous observons dans la démarche de l'IUFM de Rouen qui comporte deux étapes supplémentaires avant l'étape de situation problème.

\subsection{La démarche de L'IUFM}

La démarche pédagogique et didactique de l'IUFM de Rouen proposée lors de la formation des enseignants de la filière restauration, est fondée sur l'action de l'enseignant et de lélève; cette démarche est valable du BEP au BTS et comprend trois étapes essentielles :

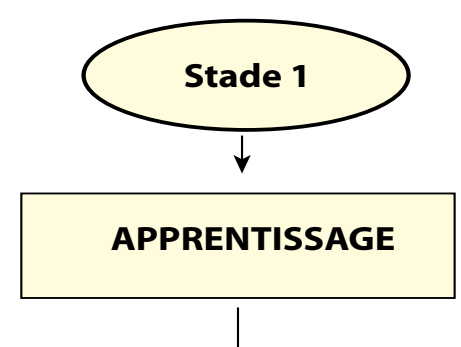

Découvrir

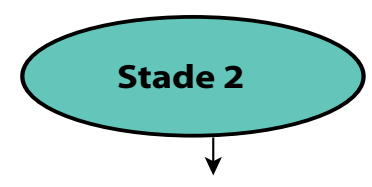

APPROFONDISSEMENT

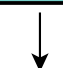

Analyser

Raisonner

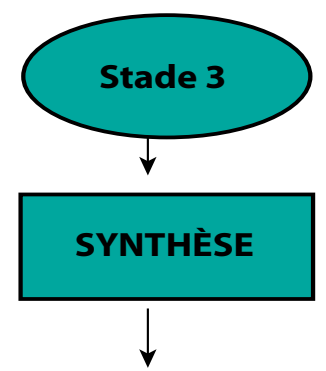

Résoudre

Figure 2 : Les stades d’apprentissage (Source: IUFM : séminaire formateur de formateur.

hra.spip.ac-rouen.fr/IMG/ppt/Seminaire_formateur_de_formateur.ppt.).

Nous observons selon la figure ci-dessus que la démarche proposée par l'IUFM de Rouen a pris en compte que les élèves, au début de leur formation professionnelle, n’ont pas la base théorique et pratique qui leur permet de travailler selon la logique de compétence qui est basée sur l'autonomie des apprenants en face d'un des problèmes ou une gamme de problèmes simultanés. Cette démarche comprend les étapes de découverte et d'analyse qui permettent aux élèves d'accumuler un corps de connaissances (théorique et pratiques) avant de passer à létape de résolution de problème. En outre, cette démarche clarifie le rôle de l'enseignant qui doit passer du rôle de transformateur et de fournisseur d'informations et de sources au rôle de concepteur et organisateur des séquences d'apprentissage.

1. Létape de découverte : l'enseignant doit en collaboration avec les élèves déterminer les objectifs de la séquence d’apprentissage et choisir un menu qui leur permet d'atteindre ces objectifs, puis il doit leur expliquer et montrer les gestes de la réalisation de la tâche que les élèves fixent en appliquant la technique. 


\section{Découvrir: stade 1}

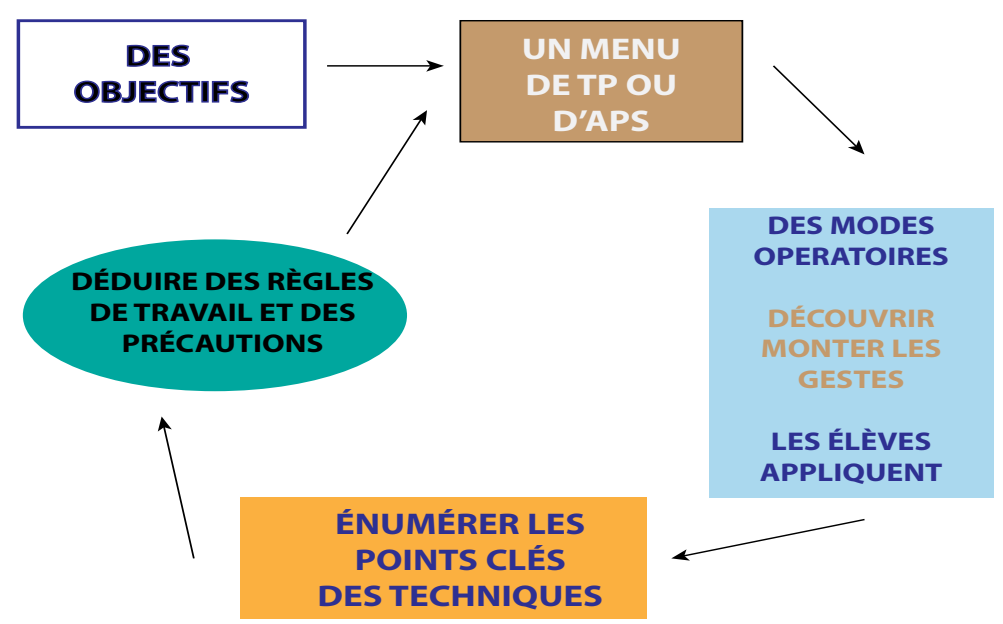

Figure $3:$ Le stade de découverte (Source: IUFM : séminaire formateur de formateur. hra.spip.ac-rouen.fr/IMG/ppt/Seminaire_formateur_de_formateur.ppt.)

2. Létape d’analyse : elle est basée sur l'intériorisation par les élèves des connaissances (théoriques et pratiques) de l’étape précédente en transformant leurs expériences antérieures accumulées en nouvelle situation d’apprentissage. Les élèves doivent analyser les résultats, évaluer les méthodes et les moyens qu'ils utilisent au cours de la séquence d’apprentissage en vue de renforcer leurs connaissances.

\section{Analyser: stade 2}

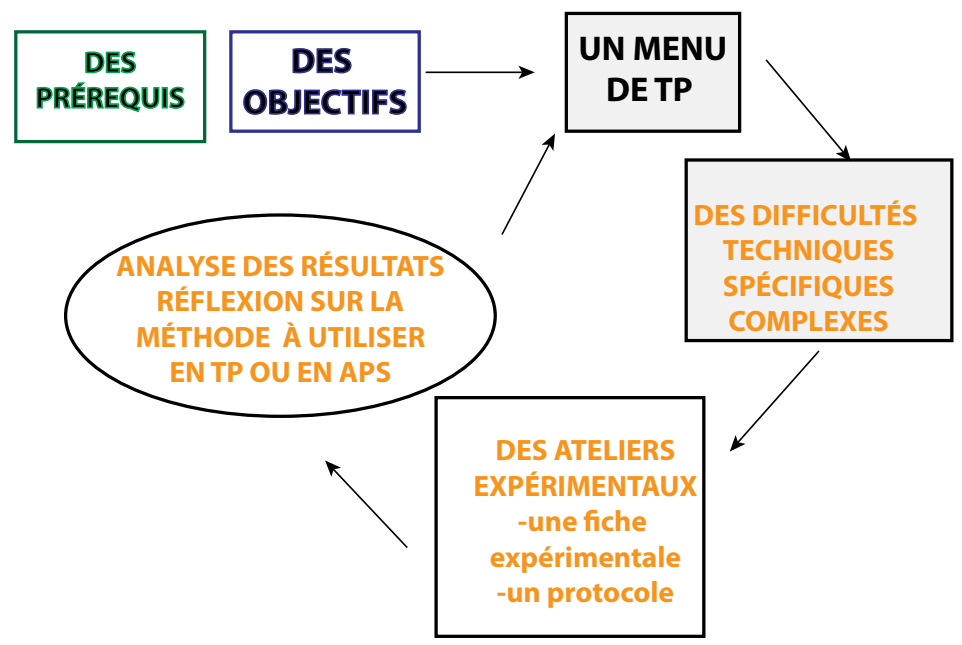

Figure $4:$ Le stade d'analyse (Source: IUFM : séminaire formateur de formateur. hra.spip.ac-rouen.fr/IMG/ppt/Seminaire_formateur_de_formateur.ppt.)

3. Létape de résolution des problèmes : elle est fondée sur le fait que les élèves deviennent capables d’appréhender, d'analyser les contraintes de production et de commercialisation, justifier l'emploi des produits retenus et apporter leur valeur ajoutée à la réalisation de manière autonome. Le rôle de lenseignant est de concevoir puis d’observer. Enfin, il doit évaluer le travail des élèves en collaboration avec eux. 


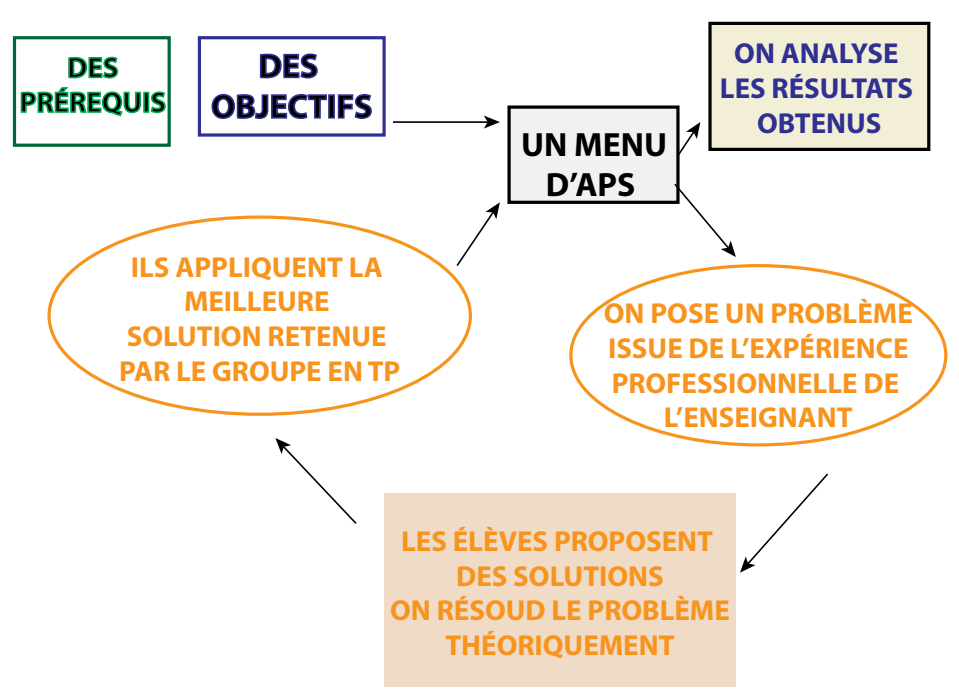

Figure 5 : Le stade de résolution d'un problème (Source : IUFM : séminaire formateur de formateur. hra.spip.ac-rouen.fr/IMG/ppt) Seminaire_formateur_de_formateur.ppt.)

Suite à l'exploration des perspectives de Guy Le Boterf et celle de l'IUFM de Rouen, nous remarquons que l'enseignant doit être un concepteur, un organisateur des situations d'apprentissage. Il doit concevoir des situations problèmes selon le niveau des élèves et leur progression.

Élaborer un plan pour résoudre le problème nécessite une réflexion sur la nature de la situation problème, sa position au cours de l'apprentissage par compétence. Ce qui nous amène à explorer la notion de situation problème dans le but de clarifier ces différentes notions.

\subsection{La situation problème}

Ballon et Dubois démontrent l'importance de la formation professionnelle qui est le principal moyen pour augmenter l'efficacité productive des salariés, on observe que l'on se limite la plupart du temps, à la formation de recettes traditionnelles et que l'efficacité de la formation ne va pas de soi. (Ballon \& Dubois, 1998). C'est pourquoi nous allons aborder le concept de situation problème en tant que moyen de développement du processus d’apprentissage. À ce stade, une clarification des différents concepts utilisés est nécessaire. La situation d'apprentissage, telle quelle est définie par Philippe Meirieu est l'ensemble des dispositifs dans lesquels un sujet s'approprie de l'information. Ces derniers peuvent être en dehors de toute structure scolaire et de toute programmation didactique. (Meirieu, 1999, p. 191). Elle est articulée sur trois fonctionnalités :

- Lanalyse de la situation, l'identification des difficultés et proposition des solutions;

- La mobilisation des outils cognitifs pour illustrer les informations théoriques et les outils métacognitifs pour analyser la démarche de résolution;

- La création d'une interface de dialogue permettant de contacter le tuteur, recevoir à l'initiative du tuteur, des conseils, des orientations (De Lièvre \& Depover, 1999, p. 284).

Selon Meirieu (1999), on distingue la situation d'apprentissage de la situation didactique qui est plutôt une situation d'apprentissage élaborée par le didacticien en fournissant les matériaux permettant de recueillir l'information et en plaçant le sujet en situation problème. Cette situation didactisée permet de faire échapper l’apprentissage à l'aléatoire, elle est construite en s’appuyant sur une triple évaluation diagnostique des motivations, des compétences et des capacités. (Meirieu, 1999, p. 191). Selon la figure ci-dessous, la relation entre les trois situations (apprentissage, didactique, problème) est réciproque : 


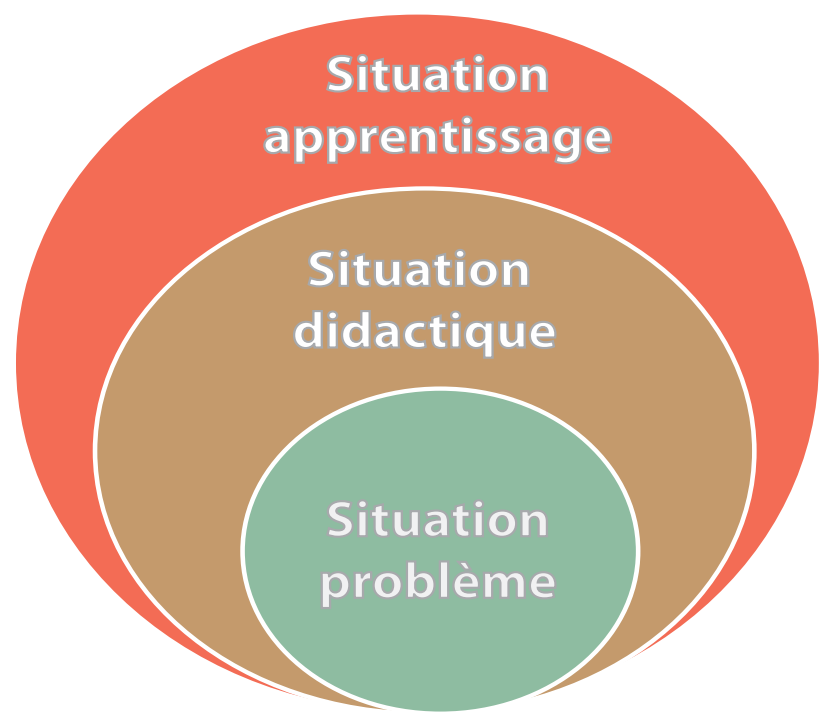

Figure 6 : La relation entre les situations de l'apprentissage.

Pour Scallon, dans une pédagogie de situation, le déroulement des activités est structuré d’après une succession de problèmes à résoudre, de tâches complexes à accomplir. Chaque problème doit faire appel à une multitude déléments appartenant au répertoire cognitif et affectif de l'élève. (Scallon, 2010, p. 34). Selon cette logique, le but est d'activer les processus mentaux et gestes chez les apprenants pour résoudre le problème en passant par la recherche des informations, l'analyse de la situation, la réalisation de la synthèse et la conception de la solution pour pouvoir transférer la solution et les résultats à d’autres situations similaires. (Scallon, 2007, p. 33-34). En s'appuyant sur les travaux de plusieurs chercheurs (CRDP, 1990; Barbier, 1996; Dalongeville \& Hubert, 2011: Morlaix 2009 et Gillet, 1997), la situation problème est une situation d'apprentissage par énigme où l'élève doit surmonter un obstacle. Sans entraînement, la mobilisation des savoirs ne peut se produire et l'enseignant dans ce cadre devient un organisateur des situations complexes qui propose des énigmes pour inciter les élèves à mobiliser leurs connaissances afin de s'acquitter de sa tâche. Pour Roland Charnay, la fonction du problème est de vérifier qu'une notion est bien assimilée par l'élève en termes de pédagogie de référence ou enseignement normatif; cette approche correspond à des problèmes fermés. Il permet aussi de tirer des situations du vécu, de motiver les élèves à travers des activités fonctionnelles en tant qu'enseignement incitatif. (Astolfi et al., 1997, p. 137-138). Ce cadre théorique montre la variété des démarches d'apprentissage selon le concept de compétence, en identifiant les changements qui affectent le rôle de l'enseignant et celui de l'élève au cours de l'apprentissage, dont le but est de s'interroger sur les démarches actuelles et leur adéquation avec le concept de compétence.

\section{Méthodologie de la recherche ${ }^{1}$}

Elle s'appuie principalement sur trois outils : questionnaire, observation et entretien.

Nous avons choisi la région lyonnaise en tant que le terrain général pour choisir léchantillon ${ }^{2}$ de notre recherche. Nous avons choisi un lycée professionnel public de la filière hôtellerie-restauration de la région lyonnaise comme terrain pour appliquer les méthodes de cette recherche qui a fait l'objet d'une convention de stage entre l'université Lyon 2 et ce lycée pendant une année scolaire du 27-09-2010 au 30-06 -2011. La population observée est constituée délèves en BEP $1^{\mathrm{e}}$ et $2^{\mathrm{e}}$ année, Bac $1^{\mathrm{e}}$ et $2^{\mathrm{e}}$ année, du corps enseignants du lycée et des restaurateurs de la région lyonnaise. Concernant le questionnaire, l’objectif principal de ce questionnaire était de savoir comment le public (élèves, enseignants et restaurateurs) de notre recherche voyaient l'interaction d'une part entre la formation initiale professionnelle et les attentes des métiers filière " restauration » et d'autre part l'orientation scolaire au collège et l'insertion professionnelle après l'obtention du diplôme afin de comparer item par item les réponses des différents acteurs de la formation. Quant aux objectifs de l'observation, ils visaient à rendre visible la démarche pédagogique appliquée en TP par les enseignants, d’observer les élèves en TP pour voir leurs difficultés lors de la réalisation de l'action, d’observer les contraintes sur le terrain qui influent sur l'acquisition des compétences et de voir s'il existe des différences entre les élèves selon leur classe BEP1; BEP2; BAC1; BAC2, concernant l’acquisition des compétences fixées dans le référentiel. Par ailleurs, lobservation a permis de relever la démarche pédagogique appliquée par le tuteur de stage et d’observer la capacité des stagiaires à transférer leurs compétences scolaires dans une situation professionnelle. La méthode de l'entretien permettait de confronter notre observation à la vision des élèves et des enseignants et de comprendre les choix pédagogiques des enseignants au regard des méthodes actuelles.

1 ANNEXE 1 Pour consulter le descriptif de la méthodologie (questionnaire, observation et entretien)

2 ANNEXe 2 Présentation synoptique de la construction des échantillons 


\section{Les résultats de la recherche}

Pour traiter la question de cet article, nous avons utilisé seulement les résultats issus de notre observation sur le terrain et de nos entretiens avec les élèves observés et leurs enseignants parce que ces deux méthodes sont étroitement liées à la thématique de cet article alors que le questionnaire concernaient les autres hypothèses de recherche : retenir la démarche appliquée par l'enseignant en TP; comparer entre la démarche de l'enseignant, celle de Guy le Boterf et de l'IUFM de Rouen; déterminer les difficultés des élèves ainsi que les contraintes sur le terrain.

\subsection{La démarche des enseignants}

Suite à notre observation au lycée dans quatre classes différentes « BEP1, BEP2, BAC1 et BAC2 » gérées par trois enseignants, nous avons observé que tous les enseignants utilisent en TP la démarche suivante :
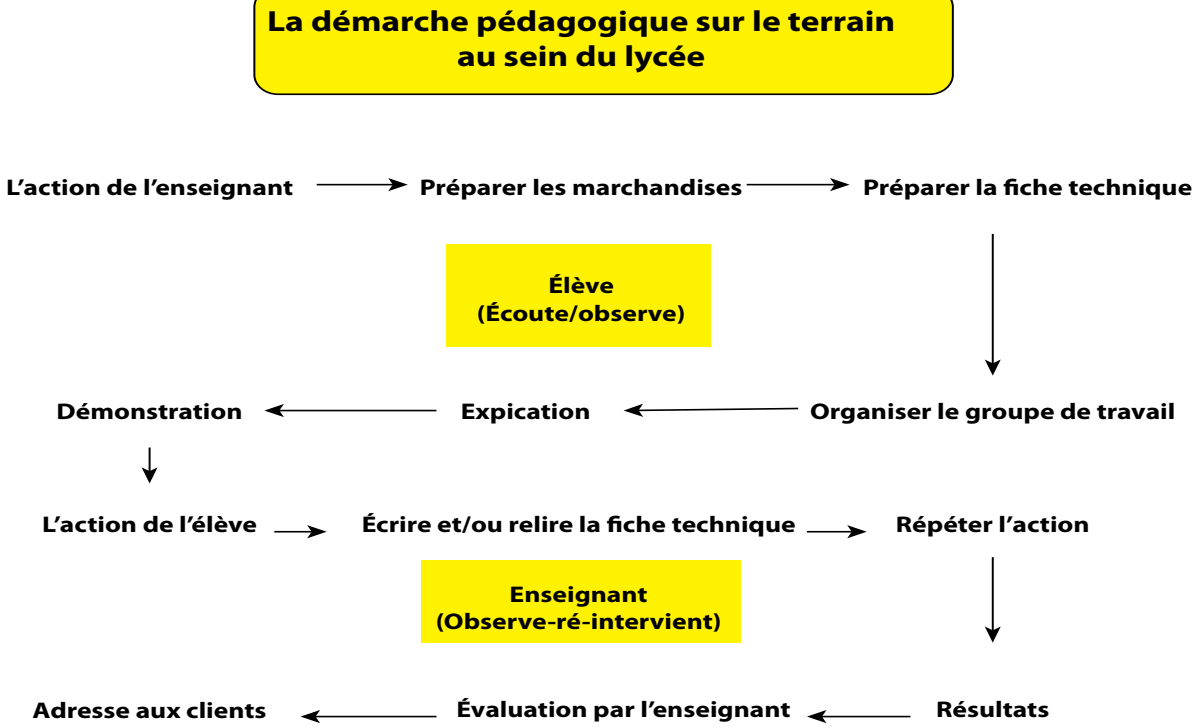

Figure 7 : Schéma de la démarche pédagogique utilisée en TP au sein du lycée professionnel « hôtellerie — restauration ».

\subsection{La comparaison entre la démarche de l'enseignant et la démarche "IUFM, Guy Le Boterf»}

Nous observons suite à l'analyse de cette démarche que l’apprentissage est fondé essentiellement sur la gestion de l'action, la réflexion, lorganisation du groupe de travail par l'enseignant d'une part et sur la répétition par les élèves d'autre part. En outre, en comparaison entre cette démarche et celle de Guy Le Boterf et/ou la démarche d'IUFM de Rouen nous observons l'absence de deux processus essentiels : l'analyse et la résolution de problème. Ce qui nous amène à nous interroger sur l'efficacité de cette démarche, les difficultés des élèves ainsi que les contraintes qui empêchent les enseignants et les élèves de passer aux étapes d’analyse et de résolution.

\subsection{Traitement et analyse des données obtenues par l'observation directe au sein de la classe et au terrain de stage : seconde phase}

À l'issue de la première phase de traitement des données d’observation, nous avons réalisé un second traitement à partir d'un affinement de la grille dobservation.

\subsubsection{La grille d’observation affinée ${ }^{3}$}

Nous avons alors établi une nouvelle grille d’observation comprenant 12 items pour repérer les points de faiblesse des élèves au regard de l'analyse du référentiel ministériel qui comprend un groupe de compétences (savoir-être, savoir, savoir-faire), de l'analyse des démarches des formateurs de l'IUFM de Rouen et de celle présentée par Guy le Boterf, mais aussi en prenant en compte les données issues de notre 
observation directe sur le terrain dans la phase exploratoire, qui ont conduit à l'analyse de la situation d'apprentissage, les discussions avec les enseignants, les élèves et le tuteur-restaurateur. Ces 12 items renvoient aux connaissances théoriques et pratiques du référentiel qui demandent à lélève d'avoir la capacité d’argumenter, de verbaliser, de réagir, prendre l'initiative dans une situation problème et transférer les compétences dans une nouvelle situation en tenant compte des interactions avec les autres membres de son équipe.

Tableau 1

Grille d'observation affinée

\begin{tabular}{|l|l|l|l|l|l|}
\hline & \multicolumn{1}{|c|}{ Items $_{\text {: }}$ critères $^{*}$} & A & B & C & D \\
\hline C1 & Savoir-être & & & & \\
\hline C2 & Savoir & & & & \\
\hline C3 & Savoir-faire & & & & \\
\hline C4 & Autonomie & & & & \\
\hline C5 & Initiative personnelle & & & & \\
\hline C6 & Raisonnement «pourquoi» & & & & \\
\hline C7 & Explication «comment» & & & \\
\hline C8 & Rapidité & & & & \\
\hline C9 & Réalisation de l'action & & & & \\
\hline C10 & Échange avec l'enseignant & & & & \\
\hline C11 & Échange entre élèves & & & & \\
\hline C12 & Transfert des compétences sur le terrain au cours du stage & & & & \\
\hline
\end{tabular}

Légende : $\grave{A}$ : tout à fait insuffisant; $B$ : plutôt insuffisant; $C$ : plutôt suffisant; $D$ : tout à fait suffisant;

* Annexe 4, Les détails des paramètres de la grille d’observation

\subsubsection{Traitement et analyse des données issues de la grille d'observation affinée concernant le terrain en TP}

Pendant une année scolaire, nous avons observé en situation 68 élèves. Cette observation a été effectuée en deux temps pour chaque élève selon que l'action a été réalisée au préalable ou non. L’objectif est de voir si les élèves sont capables de remobiliser leurs compétences théoriques et pratiques, pour réaliser une nouvelle action ou pour répéter une action réalisée antérieurement, de voir les conditions de l'acquisition des compétences en milieu scolaire et d'identifier les contraintes et les procédures de réalisation de l'action sur le terrain. Pour analyser nos données à l'aide d'ANOVA, nous avons utilisé le logiciel SPSS. Les données construites par l'observation et l'analyse sont explicitées ci-après. Nous avons effectué deux traitements pour observer la présence ou l'absence d'une différence significative suivant que l'action a été déjà réalisée ou non et en fonction des quatre niveaux scolaires (BEP1, BEP2, BAC1, BAC2).

Tableau 2

Significativité au niveau 0.05 de la comparaison des classes dans le cas « Action déjà réalisée auparavant »

\begin{tabular}{|c|c|c|c|c|c|c|c|c|c|c|c|c|c|}
\hline & \multicolumn{6}{|c|}{ Comparaison des classes } & \multicolumn{7}{|c|}{ Pourcentages (\%) Suffisant/Insuffisant } \\
\hline & & \multirow[t]{2}{*}{$\begin{array}{l}\text { BEP1 } \\
\text { BAC1 }\end{array}$} & \multirow[t]{2}{*}{$\begin{array}{l}\text { BEP1 } \\
\text { BAC2 }\end{array}$} & \multirow[t]{2}{*}{ BEP2 } & & \multirow[t]{2}{*}{$\begin{array}{l}\text { BAC1 } \\
\text { BAC2 }\end{array}$} & \multicolumn{2}{|c|}{ BEP1 } & \multicolumn{2}{|c|}{$\mathrm{BEP} 2$} & \multicolumn{2}{|c|}{ BAC1 } & BAC2 \\
\hline & & & & & & & Suf. & & Suf. & & Suf. & & Suf. \\
\hline Savoir-être & DS & DS & DS & DNS & DS & DNS & 95,7 & 4,3 & 100 & & 100 & & 100 \\
\hline Savoir & DS & DS & DS & DNS & & DNS & & 100 & 33,3 & 66,7 & & 66,7 & 100 \\
\hline Savoir -faire & DS & DS & DS & DNS & & DNS & & 100 & 52,7 & 58,3 & 59,3 & 41,7 & 54,4 \\
\hline Autonomie & DS & DS & DS & DNS & & DNS & & 100 & 25 & 75 & 29,1 & 70,9 & 23,3 \\
\hline Initiative personnelle & DNS & DNS & DNS & DNS & & DNS & & 100 & & 100 & & 100 & \\
\hline Raisonnement "pourquoi» & DS & DS & DS & DNS & & DNS & 4,4 & 95,5 & 50 & 50 & 50 & 50 & 66,7 \\
\hline Explication «comment» & DS & DS & DS & DNS & & DNS & & 100 & 50 & 50 & 50 & 50 & 66,7 \\
\hline
\end{tabular}




\begin{tabular}{|c|c|c|c|c|c|c|c|c|c|c|c|c|c|c|}
\hline Rapidité & DS & DS & DS & DNS & & DNS & 4,3 & 95,7 & 50 & 50 & 50 & 50 & 77,8 & \\
\hline Réalisation de l'action & DS & DS & DS & DNS & DS & DS & & 100 & 8,3 & 91,7 & 8,3 & 91,7 & 54,4 & \\
\hline Échange avec l'enseignant & - & - & - & - & - & - & 100 & & 100 & & 100 & & 100 & \\
\hline Échange entre élèves & DNS & DNS & DNS & DNS & & DNS & & 100 & & 100 & 95,8 & & & \\
\hline $\begin{array}{l}\text { Transfert des compétences sur } \\
\text { le terrain au cours du stage }\end{array}$ & \multicolumn{14}{|c|}{$\begin{array}{l}\text { Cette variable n'est pas traitée quantitativement parce que nous avons réussi à effectuer qu'une seule } \\
\text { observation sur le terrain au sein de l'entreprise avec un seul stagiaire. }\end{array}$} \\
\hline
\end{tabular}

Légende $: D S=$ différence significative, $D N S=$ différence nest pas significative

Tableau 3

Significativité au niveau 0.05 de la comparaison des classes dans le cas « Action jamais réalisée auparavant »

\begin{tabular}{|c|c|c|c|c|c|c|c|c|c|c|c|c|c|c|}
\hline & \multicolumn{6}{|c|}{ Comparaison des classes } & \multicolumn{8}{|c|}{ Pourcentages (\%) Suffisant/Insuffisant } \\
\hline & \multirow{3}{*}{$\begin{array}{l}\text { BEP1 } \\
\text { BEP2 }\end{array}$} & \multirow{3}{*}{$\begin{array}{l}\text { BEP1 } \\
\text { BAC1 }\end{array}$} & & \multirow{3}{*}{$\begin{array}{l}\text { BEP1 } \\
\text { BEP2 }\end{array}$} & \multirow{3}{*}{$\begin{array}{l}\text { BEP1 } \\
\text { BAC1 }\end{array}$} & & \multirow{2}{*}{\multicolumn{2}{|c|}{$\begin{array}{l}\text { BEP1 } \\
\text { BEP2 }\end{array}$}} & \multirow{2}{*}{\multicolumn{2}{|c|}{$\begin{array}{l}\text { BEP1 } \\
\text { BAC1 }\end{array}$}} & \multirow[b]{3}{*}{ Insuf } & \multirow[b]{3}{*}{ Suf. } & \multirow{2}{*}{\multicolumn{2}{|c|}{$\begin{array}{l}\text { BEP1 } \\
\text { BEP2 }\end{array}$}} \\
\hline & & & & & & & & & & & & & & \\
\hline & & & & & & & & Suf. & Insuf & Suf. & & & Insuf & Suf. \\
\hline Savoir-être & DS & DS & DS & DNS & DNS & DNS & 4,3 & 95,7 & 100 & & 100 & & 100 & \\
\hline Savoir & DS & DS & DS & DS & DS & DNS & & 100 & & 100 & & 100 & & 100 \\
\hline Savoir-faire & DS & DS & DS & DNS & DNS & DNS & & 100 & & 100 & & 100 & & 100 \\
\hline Autonomie & DS & DS & DS & DNS & DNS & DNS & & 100 & & 100 & & 100 & & 100 \\
\hline $\begin{array}{l}\text { Initiative } \\
\text { personnelle }\end{array}$ & DNS & DNS & DNS & DNS & DNS & DNS & & 100 & & 100 & & 100 & & 100 \\
\hline $\begin{array}{l}\text { Raisonnement } \\
\text { «pourquoi» }\end{array}$ & DS & DS & DS & DNS & DS & DS & & 100 & & 100 & & 100 & 21,2 & 77,8 \\
\hline $\begin{array}{l}\text { Explication } \\
\text { «comment» }\end{array}$ & DS & DS & DS & DNS & DS & DS & & 100 & & 100 & & 100 & 21,2 & 77,8 \\
\hline Rapidité & DS & DS & DS & DNS & DNS & DNS & & 100 & & 91,7 & & 95,2 & & 77,8 \\
\hline $\begin{array}{l}\text { Réalisation de } \\
\text { l'action }\end{array}$ & DNS & DNS & DNS & DNS & DNS & DNS & & 100 & & 100 & 4,2 & 95,8 & 11,1 & 88,9 \\
\hline $\begin{array}{l}\text { Échange avec } \\
\text { l'enseignant }\end{array}$ & - & - & - & - & - & - & 100 & & 100 & & 100 & & 100 & \\
\hline $\begin{array}{l}\text { Échange entre } \\
\text { élèves }\end{array}$ & DNS & DNS & DNS & DNS & DNS & DNS & & 100 & & 100 & & 95,8 & & 100 \\
\hline $\begin{array}{l}\text { Transfert des } \\
\text { compétences } \\
\text { sur le terrain } \\
\text { au cours du } \\
\text { stage }\end{array}$ & \multicolumn{14}{|c|}{$\begin{array}{l}\text { Cette variable n’est pas traitée quantitativement parce que nous avons réussi à effectuer qu'une seule observation } \\
\text { sur le terrain au sein de l'entreprise avec un seul stagiaire. }\end{array}$} \\
\hline
\end{tabular}

Légende $: D S=$ différence significative, DNS= différence n’est pas significative

Les deux tableaux ci-dessus montrent que la différence essentielle se situe entre les élèves de BEP1, d’une part, et les élèves de BEP2, BAC1 et BAC2, d’autre part. Cette différence est remarquable entre les niveaux des élèves selon qu'ils sont en train de réaliser une action déjà faite avant ou jamais faite. Nous observons aussi que la majorité des élèves témoigne de difficultés par rapport aux compétences: autonomie initiative personnelle - raisonnement pourquoi - explication comment - rapidité - réalisation de l'action, peu importe leur niveau scolaire ou que l'action soit déjà faite avant ou jamais faite.

Nous expliquons la différence entre les élèves par rapport au savoir-être en raison du fait que les élèves de BEP1 sont encore débutants, qu'ils ne connaissent pas le milieu, qu'ils ne sont pas habitués à travailler selon les méthodes mises en œuvre par l’enseignant. De plus, les enseignants sont plus rigoureux avec les élèves quant aux règles d'hygiène et aux savoir-être quaux gestes de découpe puisque la sécurité 
des clients est en jeu.

Nous expliquons la différence entre les élèves concernant les savoirs et savoir-faire, par le manque de pratique sur le terrain ou encore un faible degré de motivation comme nous avons pu le constater au travers des échanges verbaux entre l’enseignant et les élèves. Il ressort que les méthodes d'enseignement à l’ouvre semblent reposer essentiellement sur la gestion et la réflexion de l’enseignant, et la répétition par les élèves. Par ailleurs, le temps nécessaire à la confrontation des élèves aux situations problèmes dans une approche autonome n’est jamais laissé. L'intervention directe par l'enseignant se produit systématiquement dès qu'un élève se trouve en difficulté, en raison des contraintes de temps, de budget et du service des clients à midi car l'argent récolté par le restaurant permet à la direction du lycée d’effectuer les achats des matières premières nécessaires aux TP. Ces contraintes se répercutent sur l'autonomie, l'initiative personnelle, le raisonnement, l'explication, la rapidité et la réalisation de l'action des élèves : compétences non exploitées. Elles entravent également les échanges entre les élèves et leurs enseignants qui monopolisent la parole pour aller au plus vite. Ce type d’apprentissage peut également s'expliquer d'une part par des méthodes classiques d'enseignement ancrées dans la pratique des enseignants qui nont pas été initiés aux nouvelles, d'autre part en raison d'une réduction du budget essentiel aux TP dont les heures ont été diminués avec les mêmes objectifs à atteindre en fin de formation.

Nous n’avons pas pu traiter la variable C12 (transfert des compétences sur le terrain au cours du stage) quantitativement parce que nous n’avons effectué qu'une seule observation sur le terrain au sein de l'entreprise en raison du refus de nombreux restaurateurs. C'est pourquoi nous ne l'avons traitée que qualitativement. Pendant une semaine, dans l'entreprise et à travers les discussions avec le chef de cuisine, nous avons constaté que le stagiaire est incapable de réaliser l'action sans la démonstration et/ou l'explication du chef de cuisine. Ce dernier déclare ne pas faire confiance au stagiaire concernant les connaissances et les compétences professionnelles. Suite aux entretiens avec les élèves et les enseignants, il ressort que $75 \%$ des élèves déclarent que le chef a fait une démonstration et une explication préalable à la réalisation de laction par eux-mêmes. $71,42 \%$ des enseignants ont dit que le chef explique en démontrant. Ce manque de confiance des chefs des cuisines envers le stagiaire s'explique par le caractère débutant du jeune et par le coût de la marchandise ce qui justifie les difficultés à transférer les compétences scolaires dans une nouvelle situation.

Lobservation nous a permis de clarifier la méthode actuelle d'apprentissage. Cependant, la grille d’observation nous a permis plus d'identifier les difficultés rencontrées par les élèves tels que le manque de temps, d'expérience, d'autonomie ainsi que la faiblesse d'échanges entre eux. Cette méthode est basée sur le rôle de l’enseignant démonstrateur. D’une part, cette méthode n’est pas en adéquation avec la notion de compétence professionnelle, qui se concentre plutôt sur l'efficacité et la capacité de l'apprenant à agir et à résoudre des obstacles. D’autre part, elle ne correspond pas aux démarches mises en œuvre au sein de l'IUFM de Rouen, ni aux perspectives de Guy le Boterf d’où l'intérêt d'explorer nos résultats d'entretiens avec les enseignants et les élèves.

\subsubsection{Traitement et analyse des données issues des entretiens concernant le terrain en TP Entretien avec les enseignants}

En réponse à la question suivante : pensez-vous que vos méthodes d'enseignement permettent aux élèves d'acquérir les compétences dont ils auront besoin en début de carrière? Il ressort que la distribution des réponses est comme suit : $3 / 7$ oui ; $2 / 7$ jespère que oui ; $1 / 7$ je pense que oui; $1 / 7$ oui, si lenseignant fait bien son travail et si les élèves font bien leur travail.

En écho à notre remarque selon laquelle nous avions observé que dans l'atelier cuisine, le chef enseignant fait tout et les élèves ne font que répéter l'action. Nous recueillons alors les réponses qui se distribuent comme suit : 7/7 oui parce que 5/7 on n’a pas le temps en raison du service aux clients; $3 / 7$ les élèves sont débutants; et 2/7 parce que les méthodes sont directives; $2 / 7$ c'est en raison du coût des marchandises et $2 / 7$ c'est en raison du manque d'autonomie.

En réponse à la question suivante : Pensez-vous que les élèves sont capables de travailler sans l'explication, sans la fiche technique, sans la démonstration du professeur? La distribution des réponses est comme suit : 3/7 non; 3/7 des enseignants ont dit qu'ils manquent de maîtrise de base; 2/7 des enseignants ont dit qu'ils manquent de confiance de la part du professeur; 1/7 des enseignants ont dit qu'ils manquent de motivation; $1 / 7$ des enseignants ont dit que c'est impossible; $1 / 7$ des enseignants ont dit que c'est l'habitude de travail.

En réponse à la question suivante: Est-ce que le rectorat ou le ministère de l'Éducation nationale vous précise vos méthodes d’enseignement? La distribution des réponses est comme suit 3/7 des enseignants ont dit que non, on est libre; 3/7 des enseignants ont dit que oui à l'IUFM; $1 / 7$ des enseignants ont dit que c'est à travers le référentiel et le guide de l'enseignant.

En réponse à la question suivante : Pourquoi on observe que tous les professeurs utilisent les mêmes méthodes? La distribution des réponses est comme suit 3/7 des enseignants ont dit que c'est parce que c'est une méthode qui montre la technique de base; $2 / 7$ des enseignants ont dit qu'il y a d'autres méthodes; 1/7 des enseignants ont dit que c'est une méthode efficace par rapport aux contraintes: argent - temps - clients et $1 / 7$ sans réponse.

En réponse à la question suivante : Pour vous, quelle est la différence entre le concept de compétence et de qualification? La distribution des réponses est comme suit 1/7 la qualification, c'est d’avoir un CAP pour être commis ou autrement un diplôme général. La compétence c'est être capable de; 1/7 je ne sais pas, mais les élèves doivent être compétents pour réaliser certaines tâches; 1/7 La qualification est un ensemble de compétences; 1/7 CAP, il est qualifié, il va avoir des compétences sous l'autorité de quelqu'un. Le BAC, il est qualifié et c'est lui qui va acquérir des compétences; 1/7 la qualification, c'est retransmettre laction; la compétence, c'est une adaptation, c'est-à-dire on utilise une démarche différente pour arriver aux mêmes résultats; $1 / 7$ la qualification, cest un travail délicat, et la compétence cest un 
travail global; $1 / 7$ je ne sais pas; 1/7 les compétences, c’est de voir toutes les choses compétentes dans tous les domaines, les techniques; la qualification : on les a réalisées et on les maîtrise.

\section{Discussion des résultats}

Ces résultats montrent que la moitié des enseignants sont incertains de l'efficacité des méthodes d'enseignement permettant aux élèves d’acquérir les compétences dont ils auront besoin, que les élèves comptent beaucoup sur les enseignants lors de la mise en ouvre des travaux. Les enseignants justifient l'utilisation de cette méthode par le manque de temps, le caractère débutant des élèves, le coût de la marchandise et le service des clients à midi. Aussi, le rôle du MEN dans le développement des méthodes d’apprentissage est un facteur qui divise au sein des enseignants reflétant le manque de communication et déchange entre ces deux parties. De plus, les enseignants devraient varier leurs méthodes en fonction de la classe des élèves et de l'année scolaire, ce qui n'est pas le cas actuellement en raison d'une absence de coopération entre les enseignants au sein du lycée. Ces derniers estiment que la méthode actuelle est efficace parce quelle montre la technique de base aux élèves et répond aux contraintes: temps - argent - clients.

Enfin, un constat général est établi : celui de la difficulté des enseignants à faire la différence entre la notion de qualification et celle de compétence professionnelle. D’où notre interrogation sur le lien entre les programmes de formation des enseignants et les centres de recherches dans le développement des capacités des enseignants professionnels. Par ailleurs, quel est le rôle du MEN qui tend à l'introduction de nouveaux concepts, tels les compétences professionnelles, sans prendre en considération le besoin de la réhabilitation des enseignants pour ces nouveaux concepts?

\subsection{Entretien avec les élèves}

En réponse à la question suivante : Pensez-vous que les méthodes d’enseignement vous permettent d'acquérir des compétences dont vous avez besoin en début de carrière? Nous recueillons les réponses suivantes : 58/60 des élèves ont dit oui ; 1/60 des élèves ont dit non sans justification; 1/60 Avis mitigé, si on va faire gastronomie non. Associée à la réponse oui, la distribution des argumentations est comme suit 27/58 parce que ça nous permet d’avoir les bases; $13 / 58$ parce qu’on apprend les méthodes de travail pratique; $2 / 58$ Parce que ça nous facilite l'insertion professionnelle dans l'entreprise ; 2/58 mais pas assez de pratique; 1/58 ça aide mais c'est différent de l'entreprise et 15/58 sans justification.

En retour à notre remarque selon laquelle nous avions observé que dans l'atelier cuisine, le chef enseignant fait tout et les élèves ne font que répéter l'action. Nous avons recueilli les réponses suivantes : 54/60 des élèves ont dit que oui ; 1/60 des élèves ont dit que non; 5/60 des élèves sont sans réponse. Associée à la réponse oui, la distribution des réponses est comme suit : 20/54 parce qu’on est débutant sans expériences; 6/54 parce qu'on ne sait pas travailler de manière autonome; $6 / 54$ parce que le professeur a peur quon ne réussisse pas; 5/54 parce qu'on a besoin d'une démonstration; 5/54 parce qu'on doit travailler selon les méthodes et les techniques de l'enseignant; 4/54 pour qu'on fasse bien l'action; 3/54 parce qu'on a peur de prendre des risques; 3/54 parce qu'on a l'habitude de faire appel au professeur; 2/54 parce qu'il faut finir le programme et donc ne pas perdre de temps; $1 / 54$ parce le professeur est toujours derrière nous; 1/54 les élèves ne sont pas aux mêmes niveaux et 1/54 parce que c’est son métier. Sur la réponse non, la distribution des argumentations est comme suit; $1 / 1$ pas totalement, il fait une fois et nous, nous répétons.

En réponse à la question suivante : $\mathrm{Si}$ tu as un problème ou une difficulté, tu essaies de la résoudre tout seul ? La distribution des réponses est comme suit : 8/60 des élèves ont dit oui; 52/60 des élèves ont dit non. Associée à la réponse " oui », la distribution des argumentations est comme suit : $8 / 8$ des élèves ont dit qu'ils essaient de la résoudre seul, sauf s'ils n’y arrivent pas. Associée à la réponse non, la distribution des argumentations est comme suit : 8/52 parce qu'on a l'habitude de faire appel au professeur; 7/52 parce qu'on ne sait pas sans le professeur; 4/52 parce que les marchandises coûtent cher; 4/52 parce qu’on a peur de se faire disputer; $2 / 52$ parce que nous nous navons pas confiance en nous; $2 / 52$ parce qu'il est là pour nous apprendre; $1 / 52$ parce que je demande à mes amis; $1 / 52$ parce qu'on a les clients à midi; 1/52 parce qu’on n’a pas assez d'autonomie; 1/52 parce que le professeur nous montre puis il nous laisse faire et 21/52 des élèves sont sans justification.

\subsection{Discussion des résultats}

Une contradiction est notable dans les réponses des élèves puisqu'ils estiment que les méthodes actuelles sont efficaces alors qu'ils viennent affirmer nos observations en confirmant leur dépendance à l'enseignant, leur manque d'expertise, d'autonomie et d'initiative. Et, les élèves apportent les mêmes justifications que les enseignants sur les difficultés liées au terrain à savoir le manque d’expérience, le fait qu'ils soient débutants, le coût élevé de la marchandise et le manque de temps. Ces réponses peuvent sexpliquer par leur méconnaissance du référentiel et des objectifs à atteindre.

À la lumière de ces résultats et de la question fondamentale soulevée dans cet article, il est clair que lamélioration des processus d’apprentissage nécessite :

- Une clarification de lobjectif principal d'apprentissage professionnel initial car il semble y avoir une contradiction entre les enseignants et les élèves d'une part pour qui l'objectif est l'acquisition des compétences basiques et le référentiel du MEN d'autre part, qui lui met l'accent sur la nécessité de développer les capacités et les compétences des élèves à faire face au problème, d’améliorer 
l'esprit d'innovation et la créativité des élèves.

- Le ministère de l'Éducation devrait tenir compte de la nécessité pour les enseignants à suivre des formations afin de développer leurs connaissances théoriques d'une part et leurs méthodes d'apprentissage d'autre part, en particulier lors de l'inclusion de nouveaux concepts tels que la compétence professionnelle.

- Une collaboration entre les enseignants au sein du même lycée est nécessaire pour varier leurs méthodes d’apprentissage, cela permet ainsi de développer leurs connaissances théoriques et pratiques.

- Un travail de communication est nécessaire entre les IUFM, les centre de recherche d'une part et les acteurs du terrain d'autre part, afin de montrer les nouvelles méthodes d’apprentissage, leurs avantages et trouver des solutions aux difficultés au fur et à mesure de l'évolution de la profession.

- Une nécessité de définir les attentes professionnelles s'impose aux entreprises pour les apprenants et les salariés: Exécutif simplement ou exécutif — innovant - créatif et enfin autonome.

\section{Conclusion}

Nous pouvons conclure que l'enseignement, selon le concept de compétence professionnelle au cours de l'apprentissage professionnel nécessite une série de changements qui affectent le rôle de lenseignant et de lélève et les conditions topiques du processus à apprentissage ce qui conduit à la réorganisation de l'ensemble du processus de l’apprentissage.

Nous avons également observé que malgré l'introduction du concept de compétence dans le référentiel scolaire par le MEN, l'enseignant utilise la démarche classique qui repose essentiellement sur la division de travail, la démonstration, lexplication et la répétition par les élèves en vue d'accumuler les connaissances basiques.

Or, nous avons observé que l'une des difficultés des élèves sur le terrain est le manque de connaissances de base. De plus, nous avons observé que la démarche appliquée par les enseignants ne comprend pas létape d'analyse ni celle de la résolution du problème qui constituent des étapes essentielles de la démarche de L'IUFM et implicitement celle de Guy Le Boterf. Les enseignants ont justifié leur démarche par le manque de temps lié à l'activité du service à midi, le coût des ingrédients, le budget insuffisant et le manque de motivation chez certains élèves.

En outre, nous avons observé que les enseignants ont confondu le concept de qualification et le concept de compétence. Ceci nous interroge sur l'introduction du nouveau concept par le MEN sans réhabiliter au préalable le groupe d’enseignants et sur la formation continue des enseignants afin dêtre au plus près des nouvelles directives officielles. Enfin, se pose la question de la collaboration entre l'IUFM, les centres de recherche et les enseignants au cours de l'apprentissage professionnel en vue de développer la démarche des enseignants en prenant en compte les difficultés des élèves et des contraintes sur le terrain. 


\section{Références bibliographiques}

Astolfi, J.-P., Darot, E., Ginsburger-Vogel, Y. \& Toussaint, J. (1997). Mots-clés de la didactique des sciences : repères, définitions, bibliographie : Collection: Pratiques pédagogiques. Paris : De Boeck Université.

Barbier, J.-M. (1996). Les évolutions de la formation et leurs enjeux. Dans J.-C. Ruano-Borbalan, Savoir former : Bilans et perspectives des recherches sur l'acquisition et la transmission des savoirs (p. 35-46). Paris : Les éditions Demos.

Bollon, T., \& Dubois, M. (1998). Évaluation de la formation et des compétences : Quelle place pour la formation dans le cadre d'organisations apprenantes implicites? Dans J. py, \& all, Psychologie sociale et formation professionnelle : Propositions et regards critiques (p. 4961). Rennes : Presses Universitaires Rennes.

Conard, P., \& Devin, B. (2007). La professionnalisation : acquérir et transmettre des compétences. Lyon : Anact.

CRDP. (1990). Guide pour l'autoévolution de l'établissement scolaire. Lyon : Centre Régional de Documentation pédagogique.

Dalongeville, A., \& Huber, M. (2011). (Se) former par les situations problèmes : Des déstabilisations constructives : Collection : PédagogieFormation ( $2^{\mathrm{e}}$ ed.). Lyon : Chronique sociale.

De Lievre, B., \& Depover, C. (1999). Analyse des processus dans une situtation de tutorat à distance. Dans C. Depover, \& B. Noël, Lévalution des compétences et des processus cognitifs: Modèles, pratiques et contextes (p. 282-302). Bruxelles : De Boeck Université.

Direction de l'information légale et administrative de la République française. (2005). Portail Vie publique: Apprentissage et enseignement professionnel jusqu’en 2004 : sigles et définitions. Repéré à: http://www.vie-publique.fr/politiques-publiques/apprentissageenseignement-professionnel/glossaire/

ENQUÊTE BESOINS EN MAIN D’OEUVRE. (2012). Repéré le 15, 06, 2012, from Pôle emploi :

http://bmo.pole-emploi.org/?nav=secteur\&gdSecteur=6\&choixSousSecteur=IZ

FAFIH. (2011). Rapport d'activités sectoriels-hôtellerie-restauration et d'activités de loisirs. Paris : FAFIH. Repéré à :

http://www.fafih.com/sites/all/themes/fafih/uploaded_files/docs/rapport-activites-fafih.pdf

FAFIH. (2011). Rapport : Portrait sectoriel, hôtellerie-restauration et activités de loisirs-Repéré à :

http://www.fafih.com/sites/all/themes/fafih/uploaded_files/docs/Fafih-portrait-national-hotellerie-restauration-2011.pdf

Gillet, P. (1997). Construire la formation utile pour les enseignants et les formateurs, » collection pédagogie » (4e ed.).Pparis : ESF.

IUFM, d. R. (n.d.). Séminaire formateur de formateur. repéré le 07 05, 2010, à : hra.spip.ac-rouen.fr/IMG/ppt/Seminaire_formateur_de_ formateur.ppt.

Le Boterf, G. (2007). Construire les compétences individuelles et collectives (4 ed.). Paris : EYROLLES.

Meirieu, P. (1999). Apprendre.Oui, mais comment? (17 ed.). Paris : ESF.

Morlaix, S. (2009). Compétences des élèves et dynamique des apprentissages. Rennes : Presses Universités de Rennes.

Pastré, P. (2002), L'analyse du travail en didactique professionnelle, Revue Française de Pédagogie, $\mathrm{n}^{\circ} 138$, janvier-février-mars 2002 , 9-17 :

Repéré à : http://ife.ens-lyon.fr/publications/edition-electronique/revue-francaise-de-pedagogie/INRP_RF138_2.pdf.

Scallon, G. (2007). Lévaluation des apprentissages dans une approche par compétences (2 ed.). Bruxelles : De Boeck.

Scallon, G. (2010). Lévaluation formative. Bruxelles : De Boeck.

T-Kit. (2004). Les principes essentiels de la formation. Strasbourg : Conseil de l'Europe et Commission européenne. 
ANNEXE 1 le descriptif de la méthodologie (questionnaire, observation et entretien)

Le questionnaire: Nous avons établi une liste de 300 restaurants par internet dans la région lyonnaise. 217 questionnaires ont été distribués auprès des restaurateurs, 100 questionnaires distribués par voie postale, 100 distribués en face à face et 17 questionnaires distribués par les élèves. 73 questionnaires renseignés nous ont été retournés. 51 questionnaires ont été distribués auprès des enseignants en collaboration avec la direction du lycée, une fois par voie postale et deux fois par Internet, 21 questionnaires renseignés ont été retournés. Nous n’avons pas eu l'accord du rectorat pour distribuer des questionnaires auprès des enseignants des autres lycées professionnels filière «restauration» à Lyon. Enfin, sur 130 questionnaires distribués auprès des élèves 104 questionnaires renseignés ont été retournés. Le questionnaire de notre recherche comprend des questions communes pour les publics de notre recherche «enseignants, restaurateurs, élèves» et des questions spécifiques supplémentaires pour les élèves. Les questions proposées sont fermées parce qu'il est plus facile d’obtenir des réponses de la part des élèves qui ont plus de mal à exprimer leur avis face à des questions ouvertes. Par ailleurs, le temps accordé par létablissement pour les questionnaires destinés aux élèves était de 45 minutes maximum.

L'observation: notre observation s'est faite sur deux terrains: le lycée et un restaurant.

L'observation au lycée: en concertation avec la direction du lycée nous avons choisi 23 élèves BEP1, 12 élèves BEP2, 24 élèves BAC1 et 9 élèves BAC2. Nous avons suivi les élèves en TP pendant une année scolaire, 4 fois par semaine, une fois avec chaque classe. L’observation sest déroulée: les lundis, jeudis, vendredis de $8 \mathrm{~h} 00$ à $14 \mathrm{~h} 30$ et le mardi de $15 \mathrm{~h} 30$ à $22 \mathrm{~h}$.

L'observation au cours du stage en entreprise: nous avons décidé de suivre quatre élèves «BEP1, BEP2, BAC1, BAC2», mais au final, nous n’avons réussi à obtenir l’approbation que d'un seul restaurateur. Les autres restaurateurs, qui ont été contactés officiellement par le chef des travaux du lycée, ont refusé que les stagiaires soient observés dans leurs établissements.

L'entretien: nous avons mené un entretien avec chacun des 60 élèves sur 68, des classes BEP1, BEP2, BAC1, BAC2, que nous avons suivi au cours de notre observation; l'entretien a duré de 10 à 20 minutes. Nous avons mené également un entretien avec les 7 enseignants de la spécialité «technique culinaire»; l'entretien a duré de 30 à 70 minutes. Ces entretiens ont été enregistrés et transcrits manuellement par la suite. L'entretien avec les enseignants et les élèves met l'accent sur les axes suivants: l’orientation scolaire, le stage, le programme scolaire, les méthodes d’enseignement, les méthodes deévaluation, les compétences du référentiel, le recrutement de la main d’ouvre et lobjectif de la formation initiale.

\section{L'analyse de données:}

Nous avons utilisé le logiciel SPSS et le SPAD pour analyser les données récoltées par le questionnaire et lobservation en réalisant une comparaison des scores moyens entre les 3 catégories (élève, enseignant, restaurateur) par ANOVA, LSD comparaison de moyenne multiple et les pourcentages des réponses selon les modalités «Tout à fait insuffisant, Plutôt insuffisant, Plutôt suffisant tout à fait suffisante».

Les entretiens ont été transcris manuellement tout comme l’analyse des données récoltées par les entretiens a été faite. Cette analyse concerne uniquement le contenu et relève les réponses ainsi que les thématiques les plus fréquentes. Ce qui nous donne par la suite la fréquence de chaque thématique. 
ANNeXe 2 Présentation synoptique de la construction des échantillons

\begin{tabular}{|c|c|c|c|}
\hline Populations cible & $\begin{array}{l}\text { Ellêves des sections de } \\
\text { technologies culinaires des lycées } \\
\text { professionnels }\end{array}$ & $\begin{array}{l}\text { Professionnels qui enseignent } \\
\text { à ces élèves }\end{array}$ & Professionnels de la restauration \\
\hline Sous-populations cible & Région lyonnaise & Région lyonnaise & Région lyonnaise \\
\hline $\begin{array}{l}\text { Méthodes de sondage pour } \\
\text { l'enquête par questionnaire }\end{array}$ & $\begin{array}{l}\text { Tirage aléatoire d'UN lycée } \\
\text { «hôtellerie restauration » à partir } \\
\text { d'une liste de } 6 \text { établissements } \\
\text { fournie sur le site Internet du } \\
\text { rectorat de l'Académie de Lyon } \\
\text { Constitution de léchantillon } \\
\text { empirique à partir de l'ensemble } \\
\text { des élèves du lycée des niveaux } \\
\text { BEP1, BEP2, BAC1, BAC2 (le } \\
\text { niveau CAP nest pas considéré) } \\
\text { auquel est soumis le } \\
\text { questionnaire «élève » }\end{array}$ & $\begin{array}{l}\text { Tirage aléatoire dUN lycée } \\
\text { " hôtellerie restauration " } \\
\text { à partir d'une liste de } 6 \\
\text { établissements fournie sur le } \\
\text { site Internet du rectorat de } \\
\text { l'Académie de Lyon } \\
\text { Constitution de l'échantillon } \\
\text { empirique à partir de } \\
\text { l'ensemble des enseignants } \\
\text { du lycée auquel est soumis le } \\
\text { questionnaire "enseignant» }\end{array}$ & $\begin{array}{l}\text { Tirage aléatoire d'un échantillon } \\
\text { de } 100 \text { restaurateurs auxquels sont } \\
\text { envoyés les questionnaires par } \\
\text { courrier postal. } \\
\text { Tirage aléatoire de } 100 \text { restaurateurs } \\
\text { résidant dans une zone accessible } \\
\text { par les transports publics auprès } \\
\text { desquels les questionnaires ont été } \\
\text { remis en main propre et administrés } \\
\text { en face à face } \\
\text { Construction d'un échantillon } \\
\text { empirique de } 17 \text { restaurateurs } \\
\text { à partir d'une sollicitation faite } \\
\text { auprès des élèves qui ont assuré la } \\
\text { distribution des questionnaires }\end{array}$ \\
\hline $\begin{array}{l}\text { Taille des échantillons } \\
\text { auxquels le questionnaire a }\end{array}$ & 130 & 51 & 217 \\
\hline
\end{tabular}

été soumis

Méthodes de sondage pour l'enquête par observation

Taille des échantillons soumis à l'observation directe

Méthodes de sondage pour l'enquête par entretien

Taille des échantillons auprès desquels les entretiens ont été conduits
Constitution de léchantillon des 4 classes (BEP1, BEP2, BAC1, BAC2) au sein desquelles est conduite l'enquête par observation à partir de l'intervention du chef de travaux $\mathrm{BEP} 1=23 ; \mathrm{BEP} 2=12$ $\mathrm{BAC} 1=24 ; \mathrm{BAC} 2=10$

Constitution de l'échantillon des élèves sur la base du volontariat à partir de la base des 69 élèves observés

60
Constitution de léchantillon des enseignants sollicités par le chef de travaux sur la base du volontariat

3

Constitution de léchantillon des enseignants à partir de la base de 7 enseignants de la spécialité " technologie culinaire »
Constitution de l'échantillon de restaurateurs sur la base du volontariat 
ANNEXE 3 La grille d’observation

\section{Stage d'observation dans les entreprises.}

Année : 2010 - 2011

Durée : du 27 septembre 2010 au 30 juin 2011

Fiche d'observation en restauration

Classe : BEP 1 /BEP 2 /BAC 1 /BAC 2

Durée de l'observation : de à

Date : / $\quad / 2010$

\begin{tabular}{|l|l|}
\hline Rôle de lenseignant en TP & Comportement des élèves \\
\hline & \\
\hline
\end{tabular}

\section{Compétences des élèves}

\begin{tabular}{|c|c|c|c|c|}
\hline & $\begin{array}{l}\text { Tout à fait } \\
\text { insuffisant }\end{array}$ & $\begin{array}{c}\text { Plutôt } \\
\text { insuffisant }\end{array}$ & $\begin{array}{c}\text { Plutôt } \\
\text { suffisant }\end{array}$ & $\begin{array}{c}\text { Tout à fait } \\
\text { suffisant }\end{array}$ \\
\hline $\begin{array}{l}\text { Savoir-être } \\
\text { Savir }\end{array}$ & & & & \\
\hline Savoir & & & & \\
\hline $\begin{array}{l}\text { Savoir-faire } \\
\text { Autonomie }\end{array}$ & & & & \\
\hline Initiative personnelle & & & & \\
\hline $\begin{array}{l}\text { Raisonnement pourquoi } \\
\text { explication }\end{array}$ & & & & \\
\hline comment & & & & \\
\hline Réalisation de la tâche & & & & \\
\hline Echange avec l'enseignant & & & & \\
\hline $\begin{array}{l}\text { Echange entre élèves } \\
\text { Transfert des compétences sur le } \\
\text { terrain au cours du stage }\end{array}$ & & & & \\
\hline
\end{tabular}

\section{A- Les questions s'adressent aux élèves :}

1- Pouvez - vous travailler sans la fiche technique?

2- Pouvez - vous travailler sans les conseils de votre enseignant?

3- Pouvez - vous expliquer la démarche de réalisation de votre travail?

4- Pouvez — vous justifier la démarche de votre action professionnelle?

B- Les questions s'adressent aux chefs de cuisine (enseignants) :

1- Les élèves sont-ils capables de travailler sans la fiche technique?

2- Les élèves sont-ils capables de travailler sans vos conseils? 
Savoir - être :

ANNEXE 4 Les paramètres de la grille d’observation

Nombre de remarques des enseignants concernant les comportements de lélève (relation avec ses collègues et son enseignant, les règles d'hygiène, respect d'autrui).

\begin{tabular}{|l|l|l|l|}
\hline Tout à fait insuffisant & Plutôt insuffisant & Plutôt suffisant & Tout à fait suffisant \\
\hline Trois remarques & Deux remarques & Une remarque & Aucune remarque \\
\hline
\end{tabular}

Savoir : après l'explication et/ou une démonstration:

\begin{tabular}{|l|l|l|l|}
\hline Tout à fait insuffisant & Plutôt insuffisant & Plutôt suffisant & Tout à fait suffisant \\
\hline $\begin{array}{l}\text { Demande del'aide del'enseignant, } \\
\text { trois fois. }\end{array}$ & $\begin{array}{l}\text { Demande del'aide del'enseignant, } \\
\text { deux fois. }\end{array}$ & $\begin{array}{l}\text { Demande de l'aide de de } \\
\text { l'enseignant une seule fois. }\end{array}$ & $\begin{array}{l}\text { Un élève est capable de } \\
\text { comprendre et de suivre les } \\
\text { étapes des fiches techniques } \\
\text { sans l'aide de l'enseignant. }\end{array}$ \\
\hline
\end{tabular}

Savoir-faire :

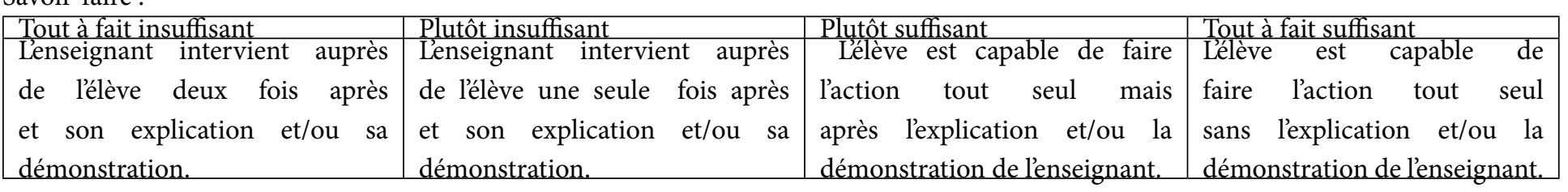

Autonomie :

\begin{tabular}{|l|l|l|l|}
\hline Tout à fait insuffisant & Plutôt insuffisant & Plutôt suffisant & Tout à fait suffisant \\
\hline Lélève est capable de faire l'action & Lélève est capable de faire laction & Lélève est capable de faire & Lélève est capable de faire \\
avec la fiche technique et les & avec la fiche technique et les & l'action sans la fiche technique & l'action tout seul sans la fiche \\
conseils et les ré-intervention & conseils et la ré-explication de & mais avec les conseils de & technique et les conseils de \\
avec démonstration pratique. & l'enseignant. & l'enseignant. & l'enseignant. \\
\hline
\end{tabular}

Initiative personnelle :

\begin{tabular}{|l|l|l|l|}
\hline Tout à fait insuffisant & lutôt insuffisant & lutôt suffisant & Tout à fait suffisant \\
\hline Dans une situation problème, & Dans une situation problème, & Dans une situation problème, & Dans une situation problème, \\
l'élève nessaie pas de la résoudre & lélève essaie de la résoudre mais & lélève essaie de la résoudre tout & lélève essaie de la résoudre \\
tout seul. & il a besoin de l'intervention de & seul mais il narrive pas seul & tout seul et il arrive. \\
& lenseignant (pratique et orale) & malgré les conseils oraux de & \\
pour la résoudre. & l'enseignant. & \\
\hline
\end{tabular}

Raisonnement pourquoi : selon les réponses des élèves à la question suivante:

Pouvez-vous justifier la démarche de votre action professionnelle?

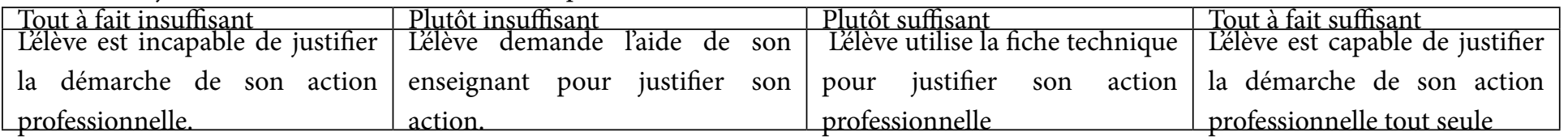

Explication comment :

Selon la réponse des élèves à la question suivante :

Pouvez-vous expliquer la démarche de réalisation de votre travail?

\begin{tabular}{|c|c|c|c|}
\hline & Plutôt insuffisant & & \\
\hline $\begin{array}{l}\text { Lélève est incapable dexpliquer } \\
\text { la démarche de son action } \\
\text { professionnelle tout seule. }\end{array}$ & $\begin{array}{l}\text { Lëlève demande laide de son } \\
\text { enseignant pour expliquer son } \\
\text { action. }\end{array}$ & $\begin{array}{l}\text { Lélève utilise la fiche technique } \\
\text { ou demande l'aide de l'un de ses } \\
\text { collègues pour d'expliquer son } \\
\text { action professionnelle. }\end{array}$ & $\begin{array}{l}\text { Lélève est capable dexpliquer } \\
\text { la démarche de son action } \\
\text { professionnelle seule. }\end{array}$ \\
\hline
\end{tabular}


Rapidité :

\begin{tabular}{|l|l|l|l|}
\hline Tout à fait insuffisant & Plutôt insuffisant & Plutôt suffisant & Tout à fait suffisant \\
\hline $\begin{array}{l}\text { Lélève a besoin de doubler le } \\
\text { temps qu'il a fallu à l'enseignant } \\
\text { pour accomplir l'action. }\end{array}$ & $\begin{array}{l}\text { Luarts a du temps qu'il a fallu } \\
\text { à l'enseignant pour accomplir } \\
\text { l'action. }\end{array}$ & $\begin{array}{l}\text { Lélève a besoin d'ajouter la moitié } \\
\text { duemps qu'il a falluà l'enseignant a besoin du même } \\
\text { pour accomplir l'action. }\end{array}$ & $\begin{array}{l}\text { temps qu'il a fallu à } \\
\text { l'enseignant pour accomplir } \\
\text { l'action. }\end{array}$ \\
\hline
\end{tabular}

\section{Réalisation de la tâche :}

En fonction des questions suivantes posées aux élèves et aux enseignants :

\section{À : les questions s'adressent aux élèves.}

1- Pouvez-vous travailler sans la fiche technique?

2- Pouvez-vous travailler sans les conseils de votre enseignant?

B : les questions s'adressent aux enseignants :

1- Les élèves sont-ils capables de travailler sans la fiche technique?

2- Les élèves sont-ils capables de travailler sans vos conseils?

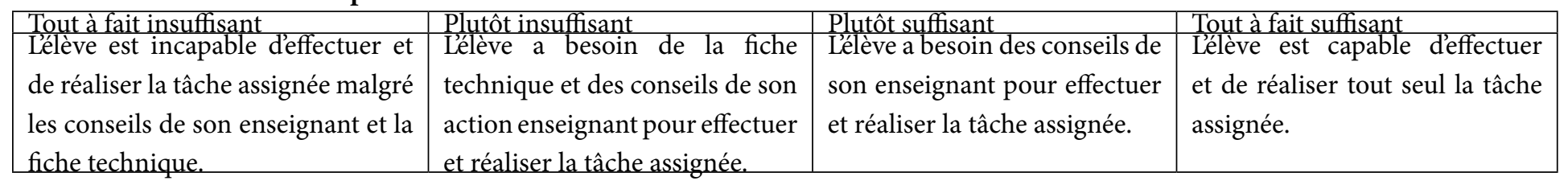

Échange avec l'enseignant :

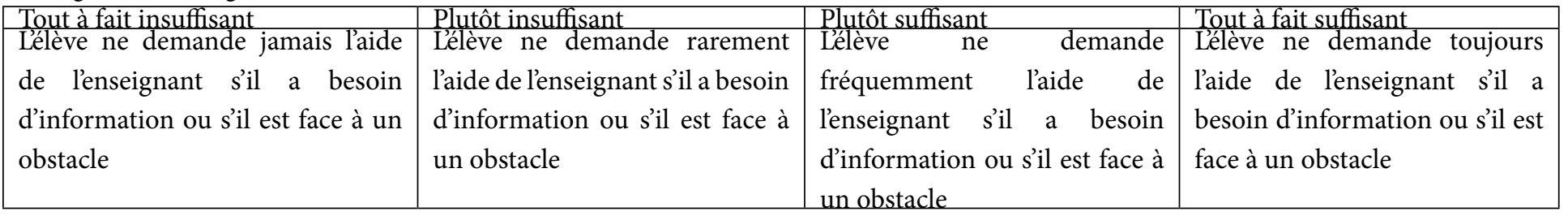

Échange entre les élèves :

\begin{tabular}{|c|c|c|c|}
\hline & & Plut & \\
\hline $\begin{array}{l}\text { Lélève ne demande jamais l'aide } \\
\text { de ses collègues s'il a besoin } \\
\text { d'information ou s'il est face à un } \\
\text { obstacle }\end{array}$ & $\begin{array}{l}\text { Lélève demande rarement l'aide } \\
\text { de ses collègues s'il a besoin } \\
\text { d'information ou s'il est face à } \\
\text { un obstacle }\end{array}$ & $\begin{array}{l}\text { Lélève demande fréquemment } \\
\text { laide de ses collègues s'il a } \\
\text { besoin d'information ou s'il est } \\
\text { face à un obstacle }\end{array}$ & $\begin{array}{l}\text { Lélève demande toujours l'aide } \\
\text { de ses collègues s'il a besoin } \\
\text { d'information ou s'il est face à } \\
\text { un obstacle }\end{array}$ \\
\hline
\end{tabular}

Transfert des compétences sur le terrain :

\begin{tabular}{|l|l|l|l|}
\hline Tout à fait insuffisant & Plutôt insuffisant & Plutôt suffisant & Tout à fait suffisant \\
\hline L’élève est incapable de transposer & Lélève demande toujours les & L'élève demande rarement les & Lélève est capable de transposer \\
seul ses compétences dans une & conseils, le suivi et l'aide du chef & conseils, le suivi du chef de & seul ses compétences dans une \\
nouvelle situation de travail, & de cuisine pour transposer ses & cuisine pour transposer ses & nouvelle situation de travail, \\
durant la période de stage, & compétences dans une nouvelle & compétences dans une nouvelle durant la période du stage \\
& situation de travail, durant la \\
& période de stage & situation de travail, durant la \\
période du stage & \\
\hline
\end{tabular}

